Tropical Journal of Pharmaceutical Research September 2021; 20 (9): 1861-1873

ISSN: $1596-5996$ (print); 1596-9827 (electronic)

(C) Pharmacotherapy Group, Faculty of Pharmacy, University of Benin, Benin City, 300001 Nigeria

\title{
Evaluation of antioxidant activation by potential nuclear factor (erythroid-derived 2)-like 2 (Nrf2)/Keap1 complex inhibitors
}

\author{
Inas Saleh Almazari ${ }^{1 *}$, Shada Youssef Elhayek ${ }^{2}$ \\ ${ }^{1}$ Faculty of Pharmacy, Department of Clinical Pharmacy, Zarqa University, Zarqa 13132, Jordan, ${ }^{2}$ Faculty of Mathematical and \\ Natural Sciences, Department of Pharmacy, Heinrich-Heine-University Düsseldorf, 40522 Düsseldorf, Germany
}

*For correspondence: Email: ialmazari@zu.edu.jo; Tel: 0096253821100 ext 1816; Fax: 0096253821120

Sent for review: 26 July 2020

Revised accepted: 16 August 2021

\begin{abstract}
Purpose: To investigate the binding affinities of forty-one (41) National Cancer Institute (NCl)generated compounds, to the Nrf2 ligand, and possible activation of Nrf2 in the MCF-7 cell line.

Methods: To investigate the inhibition of the Nrf2/Keap1 complex, the MCF-7 cell line was treated with each of the 41 compounds, at a working concentration of $30 \mu \mathrm{M}$. The extent of Nrf2 activation and corresponding Nrf2/Keap1 complex inhibition was evaluated in terms of Nrf2 expression and its antioxidant-associated enzyme gamma-glutamylcysteine synthetase (GCS), using western blot analysis.

Results: Twenty-nine compounds out of the 41 targeted compounds activated GCS, and some showed comparable or greater activation capacity than the standard Nrf2 activator $t B H Q$. To confirm that the activation of GCS was mediated via Nrf2 activation, cell lysates were tested for their Nrf2 protein expression, and it was found that Nrf2 was activated by the examined compounds for more than $24 \mathrm{~h}$, indicating that the effect of the chosen compounds were not transient.

Conclusion: These results might be useful for identifying better targets for cytoprotection, and for oxidative stress alleviation through Nrf2 pathway activation. Further studies are required on the effects of these targets on the prevention and treatment of various oxidative stress disorders, including cancer.
\end{abstract}

Keywords: NCl compounds, Nrf2/Keap1, Cancer, Antioxidants, Gamma-glutamylcysteine synthetase (GCS), MCF7, Pharmacophore, Docking, Co-crystallized structure

\begin{abstract}
This is an Open Access article that uses a funding model which does not charge readers or their institutions for access and distributed under the terms of the Creative Commons Attribution License (http://creativecommons.org/licenses/by/4.0) and the Budapest Open Access Initiative (http://www.budapestopenaccessinitiative.org/read), which permit unrestricted use, distribution, and reproduction in any medium, provided the original work is properly credited.

Tropical Journal of Pharmaceutical Research is indexed by Science Citation Index (SciSearch), Scopus, International Pharmaceutical Abstract, Chemical Abstracts, Embase, Index Copernicus, EBSCO, African Index Medicus, JournalSeek, Journal Citation Reports/Science Edition, Directory of Open Access Journals (DOAJ), African Journal Online, Bioline International, Open-J-Gate and Pharmacy Abstracts
\end{abstract}

\section{INTRODUCTION}

Cancer targeted therapy has developed immensely through targeting small molecules, which upon inhibition or activation, lead to cancer prevention or treatment. Nuclear factor erythroid 2-related factor 2 (Nrf2) is a basic DNA-binding leucine zipper transcription factor. The erythroid- derived 2-like2 gene (NFE2L2) encodes a protein that regulates cellular stress-induced metabolic and proteotoxic DNA damage, thereby helping cells to cope with stress-induced injury [1,2]. Moreover, Nrf2 is a defensive transcription factor that mitigates oxidative stress-induced inflammation [3]. It regulates the expression of antioxidant response elements (AREs) which 
modulate the expressions of numerous antioxidant enzymes, primarily GCS, as part of the inflammation-control cascade. The enzyme GCS protects cells against inflammation-induced oxidative damage by activating additional downstream pathways $[3,4]$. Several drugs that stimulate the NFE2L2 pathway have shown promising potential as new treatments for solid tumors such as thyroid and ovarian carcinomas [5].

Under resting conditions, Nrf2 is retained within the cytoplasm by complexing with Keap1 and Cullin3 (CUL3). The short half-life of Nrf2 (20 min) may be due to its continuous ubiquitination by Keap1 and CUL3 through regulation of proteasomal degradation of Nrf2. On the other hand, during oxidative stress, Nrf2 proteasomal degradation stops, and Nrf2 migrates to the nucleus and binds its DNA promoter. This is due to the disruption of critical cysteine residues in Keap 1, which ends up disrupting the ubiquitination process [5]. When Nrf2 is not ubiquitinated, it accumulates inside the cytoplasm and undergoes nuclear translocation where it combines with a small Maf protein to form a heterodimer which binds to AREs within the upstream promoter region of numerous antioxidative genes to initiate their transcription [6]. For that reason, the inhibition of Nrf2/Keap1 complex which finally ends up in Nrf2 activation, is a potential key goal in cancer prevention and therapy [5].

Considering the promising results of targeting the Nrf2/Keap1 complex in chemoprevention with antioxidant and anti-inflammatory drugs, studies on chemical substances with potential to bind and inhibit the Nrf2/Keap1 complex may be useful for identifying new potential chemoprotective agents.

The aim of the present study was to create compounds with high-affinity binding to Nrf2, using structure-based modeling and docking systems. Then, the expressions of Nrf2 and its housekeeping effector enzyme GCS were assayed in MCF7 cell line (a human breast cancer cell line) after treatment with 41 chosen compounds with various functional groups but with high binding affinities to Nrf2.

\section{EXPERIMENTAL}

\section{Molecular modeling}

A previously published in-house modeling station was used to map pharmacophores related to Nrf2 protein [7].

\section{Pharmacophore generation from co- crystallized structure}

The pharmacophoric features of Nrf2 were investigated using a 4L7B co-crystallized structure [7]. The 3D coordinates of Nrf2 were recovered within the Protein Data Bank (Nrf2 PDB code: 4L7B, resolution: $2.4 \AA$ ) [8]. The DS 4.5 templates were used to feature hydrogen atoms into the protein residues. The parameters used to generate the receptor-ligand pharmacophore within DS 4.5 were set to get minimum quantity of the four features, and a maximum of ten. The resultant pharmacophores modeled were 10 in number, with co-crystallized water remaining inside the protein structure. The selective pharmacophore models based entirely on receptor-ligand interactions were created using Accelrys DS v4.5 and Receptor-Ligand Pharmacophore Generation Protocol with default settings. The following set of specified binding ligand properties is recognized: hydrogen bond acceptor (HBA), hydrogen bond donor (HBD), hydrophobic (Hbic), negative ionizable (NI), positive ionizable $(\mathrm{PI})$, and ring aromatic (RA) [7, $9,10]$.

\section{Molecular docking}

Molecular docking was implemented within DS 4.5 so as to assign Gasteiger-Marsili charges to the protein atoms [9]. The required docking conditions retained the intrinsic water molecules. The subsequent docking experiments utilized protein construction without energy minimization. The docking parameters in LigandFit algorithm consider the ligand to be flexible while selecting the receptor's stiffness $[11,12]$. The binding site was constructed and the selected hits were docked into it using co-crystallized ligand (1VV701 in Nrf2, 4L7B, resolution: $2.41 \AA$ ) in the presence of the indicated water molecules, under the following docking arrangements: Monte Carlo search parameters were set to 15000 trials, and attempts for rotations with polar hydrogen equalled $30.0^{\circ}$, the Root Mean Square threshold for ligand-to-binding-site shape matching was established at $2.0 \AA$, with a maximum of 1.0 binding-site partitions, and an electric grid that stretched $3.0 \AA$ from the binding place was employed. DS 4.5 was used to investigate protein-ligand interactions which were assessed using consistent force field (v.1.02) and a distance-dependent dielectric with a non-bonded cutoff distance of $10.0 \AA$. The interaction energies were calculated using soft potential energy estimates and a trilinear interpolation value. For each docked ligand orientation, the rigid body ligand minimization parameters were 20 steepest descent iterations followed by 40 
Broyden-Fletcher-Goldfarb-Shanno minimization iterations. The energy within the binding site of the best 10 poses was subsequently reduced to a maximum of 200 stiff body repetitions [9].

\section{Pharmacophore mapping}

The value of fit for any drug is determined by the total mapped hypothesis features which reflect the total pharmacophore features superimposed on the analogous chemical moieties inside the fitted molecule. To match the mapped features with the docked posture, the screened National Cancer Institute $(\mathrm{NCl})$ hits were matched with the virtual co-crystallized Nrf2 pharmacophore model 4L7B_2_03 using the "best fit" option inside CATALYST in DS 4.5 [10]. Then, the rigid-body docking feature was used to attach mapped compounds in the binding region of Nrf2 $[7,13]$.

\section{Reagents and chemicals used for cell culture assay}

The National Cancer Institute ( $\mathrm{NCl}$, USA) was the source of the forty-one compounds, each with a specific Cancer Chemotherapy National Service Center (NSC) number. The other reagents were tert-butylhydroquinone (tBHQ; 30 $\mu \mathrm{M})$ as positive control; dimethylsulfoxide (DMSO), double distilled water, Roswell Park Memorial Institute (RPMI) media for cells, heatinactivated fetal bovine serum (FBS), TrypsinEDTA, L-glutamine, Dulbecco's phosphatebuffered saline (PBS), streptomycin, and penicillin were purchased from Capricorn Scientific $\mathrm{GmbH}$ (Ebsdorfergrund, Germany). Dithiothreitol (DTT) and bovine insulin were products of Sigma Chemical Co. (St Louis, MO). Radioimmunoprecipitation assay (RIPA) lysis buffer was purchased from Cell signaling (Beverly, USA). Primary antibodies for Nrf2, GCS, and $\beta$-actin, and secondary antibodies were obtained from Santa Cruz Biotechnology (Santa Cruz, CA), while Amersham ECL detection reagent was provided by $\mathrm{GE}$ Healthcare (Buckinghamshire, UK). Bicinchoninic acid (BCA) protein test reagent was purchased from Pierce Biotechnology (Rockford, IL). Pall Gelman Laboratory (Ann Arbor, MI) provided polyvinylidene difluoride (PVDF) membranes. All other chemicals were either analytical or of the highest purity grade available.

\section{Preparation of the tested compounds for in vitro assay}

The $\mathrm{NCl}$ compounds were supplied as dry powders weighing 5-10 mg. The compounds were solubilized in DMSO to make $0.02 \mathrm{M}$ stock solutions. Each stock solution was diluted to final working concentration of $30 \mu \mathrm{M}$ which was used in assay of protein expression levels of GCS and Nrf2 in the cell line studied.

\section{Cell culture}

The MCF7 cells were grown in RPMI medium supplemented with $10 \%$ FBS, $1 \%$ penicillinstreptomycin, $1 \%$ glutamate, and $10 \%$ insulin $(10 \mu \mathrm{g} / \mathrm{mL})$. The cells were treated for $24 \mathrm{~h}$ at 37 ${ }^{\circ} \mathrm{C}$ with $\mathrm{tBHQ}$, DMSO, or $\mathrm{NCl}$ compounds, in a humidified environment containing $5 \% \mathrm{CO}_{2}$ and $95 \%$ air. Thereafter, the cells were lysed to get the whole lysate protein which was used for western blot assay.

\section{Western blot analysis}

The cells were washed two times with PBS after drug treatment and then detached on ice with $1 \mathrm{x}$ RIPA lysis buffer supplemented with $1 \%$ phenylmethylsulfonyl fluoride for $1 \mathrm{~h}$. After cell detachment, the cell lysates were centrifuged at $17,000 \mathrm{~g}$ for $15 \mathrm{~min}$. Total protein contents of the supernatants were determined using BCA reagent. Equal amounts of protein $(30-50 \mu \mathrm{g})$ were resolved using sodium dodecyl sulfate (SDS)-polyacrylamide gel electrophoresis, followed by transfer to PVDF membranes. After blocking for $1 \mathrm{~h}$ at room temperature (RT) with 5 $\%$ fat-free dry milk-PBST (Tris-buffered saline containing $0.1 \%$ Tween-20) buffer, the membranes were incubated overnight with specific primary antibodies diluted in PBS $(1: 1000)$ or $3 \%$ fat-free dry milk-PBS. The blots were washed thrice with PBST, prior to incubation for $1 \mathrm{~h}$ at RT with horseradish peroxidase-labelled secondary antibodies diluted $1: 2000$ in $3 \%$ fat-free dry milk-PBS. Then, blots were washed and incubated for 1 min with ECL Prime Western Blotting Detection Reagent, in accordance with the manufacturer's instructions. The images were observed with ultrasensitive chemillumences Western blot image model FCE (Proteinsimple $\AA$ ).

\section{RESULTS}

Table 1 displays the generated pharmacophores using DS studio 4.5. The Nrf2 protein cocrystallized with 1VV701 (4L7B, resolution 2.41 $A)$ was used to generate the pharmacophores. 4L7B_2 03 was selected because of high ROCAUC $>80 \%$ and the three diverse features; HBA, Hbic, and RA (Table 1). The 4L7B_2_03 (Tables 2 and 3; Figure 1A) was used as a search tool for selecting 534 compounds out of the 257,000 compounds in the $\mathrm{NCl}$ database. The chosen compounds were categorized based on their fit values, and the top 41 were evaluated in vitro for 
GCS and Nrf2 activation using western blot analysis.

Figure 2 shows the potential interaction of cocrystallized structure (1VV701) inside the binding pocket of Nrf2 (PDB: 4L7B, resolution: $2.41 \AA$ ) after $2 \mathrm{D}$ analysis using the DS 4.5 visualizer. The results revealed the co-crystallized compound (1VV701) inside the binding region of Nrf2. The equivalent amino acids involved in hydrogen bond interactions were Arg415, Asn414, and Asn387; as well as those involved in Van der Waal and hydrophobic interactions i.e., Ala556, Gly364, Gly509, Tyr334, and Tyr572. These amino acids created the binding pocket for the suitable co-crystallized ligand (1VV701). The mapping properties of 4L7B_2_03 (Figure $1 \mathrm{C}$ ) can be explained based on the following mappings: the isoquinoline group was mapped with two hydrophobic features that correlated with Van der Waal interactions with hydrophobic portions of Gly364, Gly509, and Ala556; the interaction of phenyl groups with electron-rich phenyl groups of Tyr334 and Tyr572 was mapped with the isoindole group and the aromatic ring group.

Table 1: Pharmacophores co-crystallized with Nrf2 structure (4L7B)

\begin{tabular}{llcccc}
\hline Pharmacophore & Model & $\begin{array}{c}\text { Number of } \\
\text { features }\end{array}$ & Feature set & $\begin{array}{c}\text { Selectivity } \\
\text { score }\end{array}$ & ROC-AUC* $^{\#}$ \\
\hline 4L7B_2_01 & Pharmacophore_01 & 4 & HHNR & 8.58 & 0.65 \\
4L7B_2_03 & Pharmacophore_02 & 5 & AAHHR & 7.88 & 0.50 \\
4L7B_2_03 & Pharmacophore_03 & 4 & AHHR & 6.37 & 0.833 \\
4L7B_2_03 & Pharmacophore_04 & 4 & AHHR & 6.37 & 0.53 \\
4L7B_2_05 & Pharmacophore_05 & 4 & AAHR & 6.37 & 0.56 \\
4L7B_2_06 & Pharmacophore_06 & 4 & AAHR & 6.37 & 0.61 \\
4L7B_2_07 & Pharmacophore_07 & 4 & AAHH & 6.37 & 0.88 \\
\hline
\end{tabular}

*A: HBA, N: NI, H: Hbic, R: RA; \# Validation parameter using the default method

Table 2: Features of pharmacophores and corresponding weights, tolerances, and 3D coordinates of generated pharmacophore from co-crystallized Nrf2 (4L7B)

\begin{tabular}{|c|c|c|c|c|c|c|c|c|}
\hline \multirow[t]{3}{*}{ Model } & \multirow{3}{*}{$\begin{array}{l}\text { Definition } \\
\text { Tolerances }\end{array}$} & \multicolumn{7}{|c|}{ Chemical feature } \\
\hline & & & \multicolumn{2}{|c|}{$H B A$} & \multirow{2}{*}{$\begin{array}{c}\text { Hbic1 } \\
1.6\end{array}$} & \multirow{2}{*}{$\begin{array}{c}\text { Hbic2 } \\
1.6\end{array}$} & \multicolumn{2}{|c|}{$R A$} \\
\hline & & & 1.6 & 2.2 & & & 1.6 & 2.2 \\
\hline \multirow[t]{3}{*}{ 4L7B_2_03 } & & $X$ & 1.13 & 1.27 & -3.4 & -3.2 & -6.1 & -9.0 \\
\hline & Coordinates & Y & 2.49 & 5.49 & 3.2 & 6.6 & 0.73 & 1.42 \\
\hline & & Z & -26.78 & -26.6 & -30.4 & -30.5 & -24.6 & -24.6 \\
\hline
\end{tabular}

Table 3: Exclusion spheres added to the generated pharmacophore

\begin{tabular}{lcccc}
\hline Feature & $\mathbf{X}$ & $\mathbf{Y}$ & $\mathbf{Z}$ & Radius \\
\hline ExcludedVolume_1.7 & 0.791 & 6.677 & -29.766 & 1.724 \\
ExcludedVolume_1.8 & -9.632 & 0.62 & -19.707 & 1.008 \\
ExcludedVolume_1.9 & -9.72 & -2.9 & -22.076 & 1.27 \\
ExcludedVolume_1.10 & 1.385 & -3.711 & -26.942 & 1.27 \\
ExcludedVolume_1.11 & 0.33 & 3.264 & -34.284 & 1.27 \\
ExcludedVolume_1.12 & -9.042 & -2.722 & -27.925 & 1.27 \\
ExcludedVolume_1.13 & 3.226 & 3.269 & -30.447 & 1.154 \\
ExcludedVolume_1.14 & -1.371 & 11.268 & -31.555 & 1.27 \\
ExcludedVolume_1.15 & -6.944 & 0.323 & -32.432 & 1.368 \\
ExcludedVolume_1.16 & -9.375 & 6.394 & -29.948 & 1.27 \\
ExcludedVolume_1.17 & 0.11 & -0.286 & -33.055 & 1.368 \\
ExcludedVolume_1.18 & -9.81 & 1.808 & -25.816 & 1.6 \\
ExcludedVolume_1.19 & 4.726 & 0.095 & -27.469 & 1.368 \\
ExcludedVolume_1.20 & -7.614 & -3.791 & -20.923 & 1.008 \\
ExcludedVolume_1.21 & -4.482 & 1.825 & -34.727 & 1.27 \\
ExcludedVolume_1.22 & -3.501 & -1.24 & -19.984 & 1.454 \\
ExcludedVolume_1.23 & -6.96 & -0.755 & -18.968 & 1.27 \\
ExcludedVolume_1.24 & -5.507 & 12.055 & -28.748 & 1.27 \\
ExcludedVolume_1.25 & -3.843 & -3.714 & -28.858 & 1.664 \\
ExcludedVolume_1.26 & -7.144 & 5.859 & -31.832 & 1.154 \\
ExcludedVolume_1.27 & -6.2 & 10.26 & -31.61 & 1.27 \\
\hline
\end{tabular}




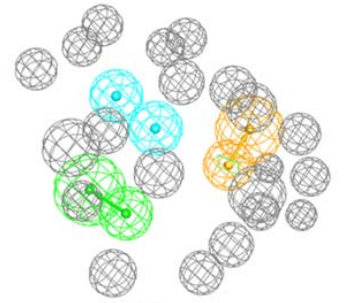

(A)

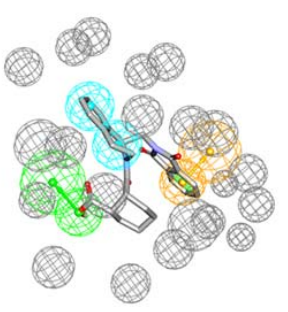

(C)

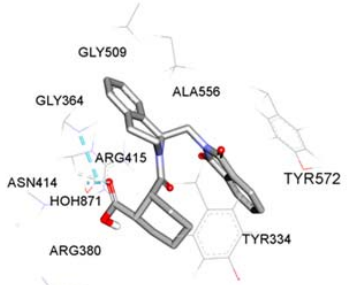

(B)

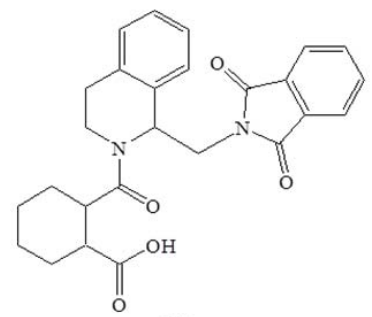

(D)
Figure 1: HBA (green vectored two spheres), Hbic feature (blue sphere), RA (orange vectored two spheres), and exclusion volume (gray spheres). (A) Hypo4L7B_2_03, the best pharmacophore generated by DS studio 4.5; (B) 1VV701 co-crystallized in Nrf2 (4L7B, resolution: 2.41 A), (C). Pharmacophore mapping using co-crystallized Ligand 1VV701, (D) 1VV701 chemical structure.

Carboxylic hydroxyl group was mapped with hydrogen bond acceptor feature which matched hydroxyl group interaction with proton acceptor guanidino groups in Arg414 and Arg380 (Figure $1 \mathrm{~B})$. The co-crystallized posture of 1VV701 within the binding pocket of Nrf2 (PDB: 4L7B, resolution: $2.41 \AA)$ corresponded with the mapping of 1VV701 with 4L7B_2_03 (Figure 1 C).

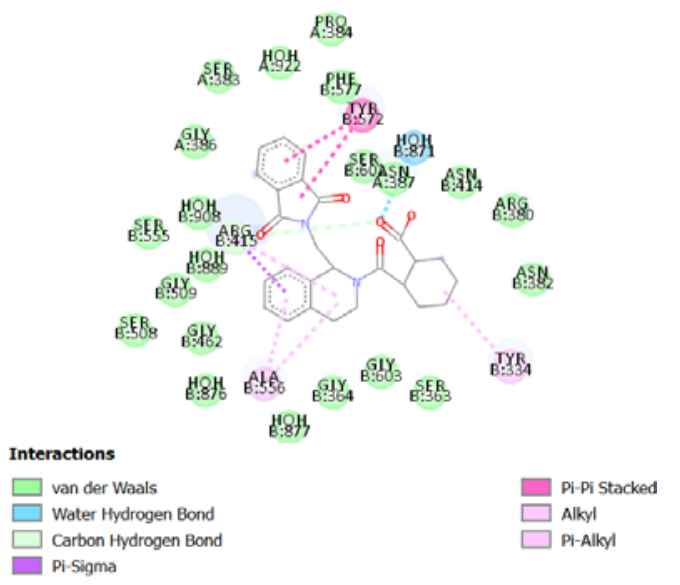

Figure 2: The two-dimensional study of co-crystallized 1VV701 at the Nrf2 binding site, as performed using the DS 4.5 visualizer (4L7B, resolution: 2.4 )

In in vitro investigation of the $\mathrm{NCl}$ compounds, MCF7 cells were treated with DMSO (negative control), tBHQ (positive control) [14], or the 41 $\mathrm{NCl}$ selected compounds, for $24 \mathrm{~h}$ (Figure 3 to $7)$. The effects of the selected compounds on protein expressions of the antioxidant enzymes i.e., heme oxygenase-1, $\mathrm{NAD}(\mathrm{P}) \mathrm{H}$ quinone oxidoreductase-1, and GSC were determined to check if the target compounds upregulated the cytoprotective pathway. The expression of GCS was chosen as representative antioxidant in the testing of all the compounds after MCF7 treatment for $24 \mathrm{~h}$. At least 6 out of the 41 compounds induced GCS expression at least as much as $\mathrm{tBHQ}$ after treatment for $24 \mathrm{~h}$, as shown in Figure 7. To confirm the results obtained, the experiments were repeated independently at least three times.

To verify if the expression of GCS was mediated via Nrf2 pathway activation, the expression of Nrf2 in MCF7 cells was assayed after treatment with either DMSO, tBHQ, or NSC compounds (Figure 10). The results showed that Nrf2 protein expression was upregulated by most of the tested compounds (29 out of 41) as much as $t B H Q$, indicating that GCS expression was upregulated through the Nrf2 pathway (Figure 10).

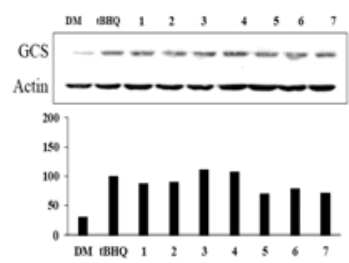

(A)
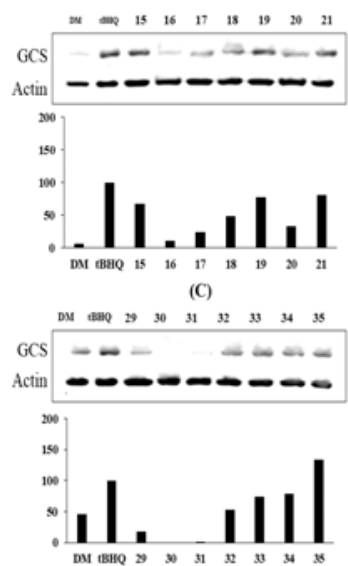

(E)

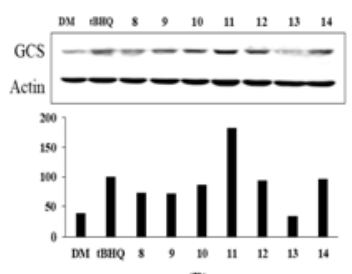

(B)

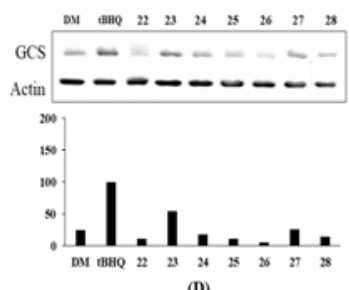

(D)

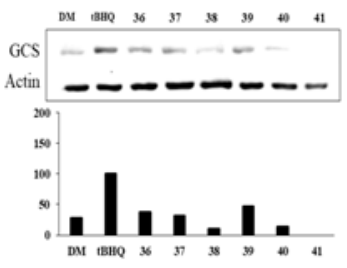

(F)
Figure 4: Effect of Nrf2/Keap1 complex inhibitors on GCS protein expression in MCF7 cells. The MCF7 cells were treated for $24 \mathrm{~h}$ with DMSO (DM), $30 \mu \mathrm{M}$ tBHQ, or $30 \mu \mathrm{M}$ of each of NSC compounds 1-7 (A); 8$14(B), 15-21(C), 22-28(D), 29-35(E)$, and 36-41 (F). Western blot analysis was used to assay the expressions of GCS and Nrf2, with F-actin as loading control

Trop J Pharm Res, September 2021; 20(9): 1865 


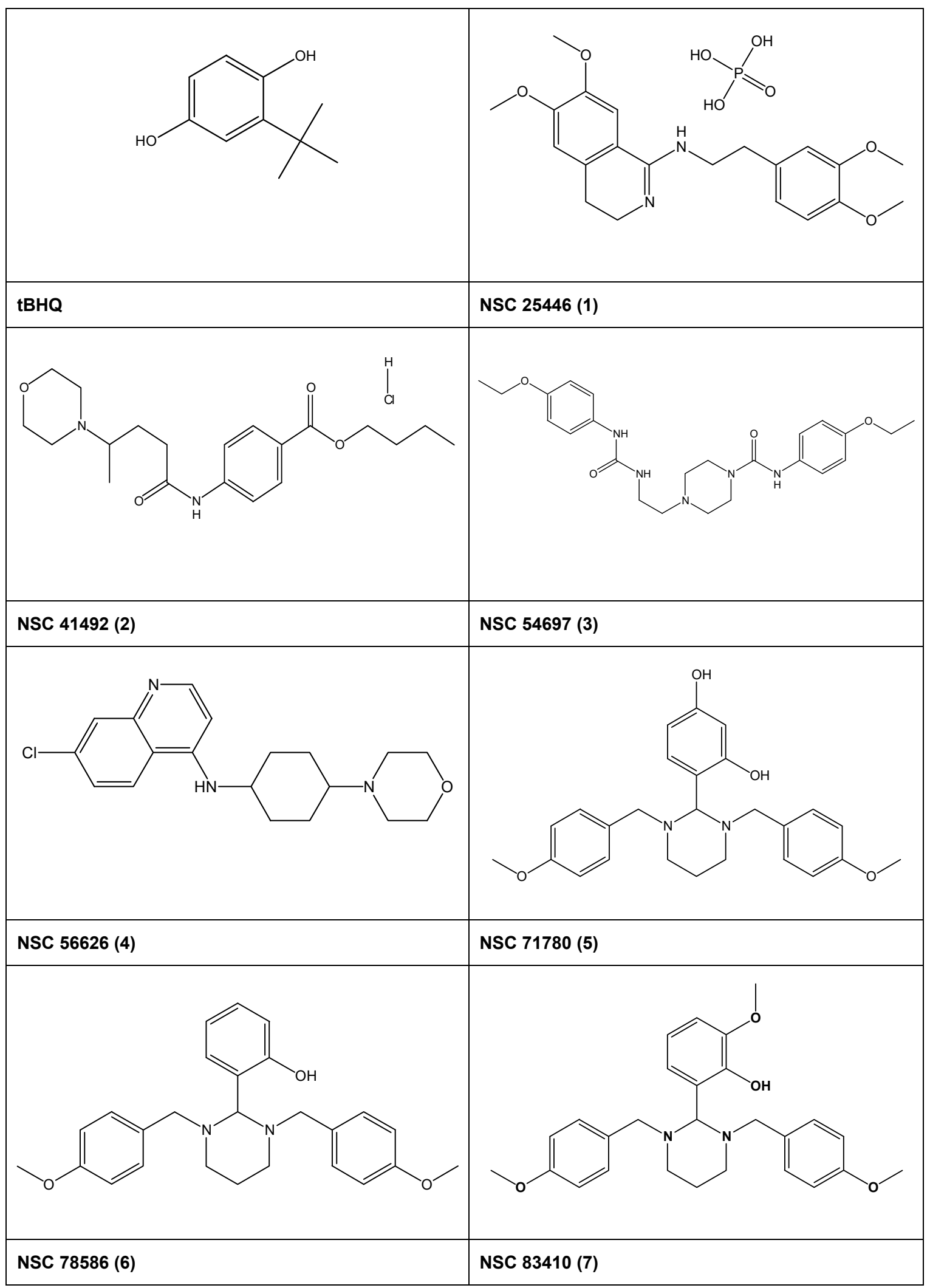

Figure 3: Chemical structures of $\mathrm{tBHQ}$ and NSC compounds (1-7) selected for Nrf2 activity 


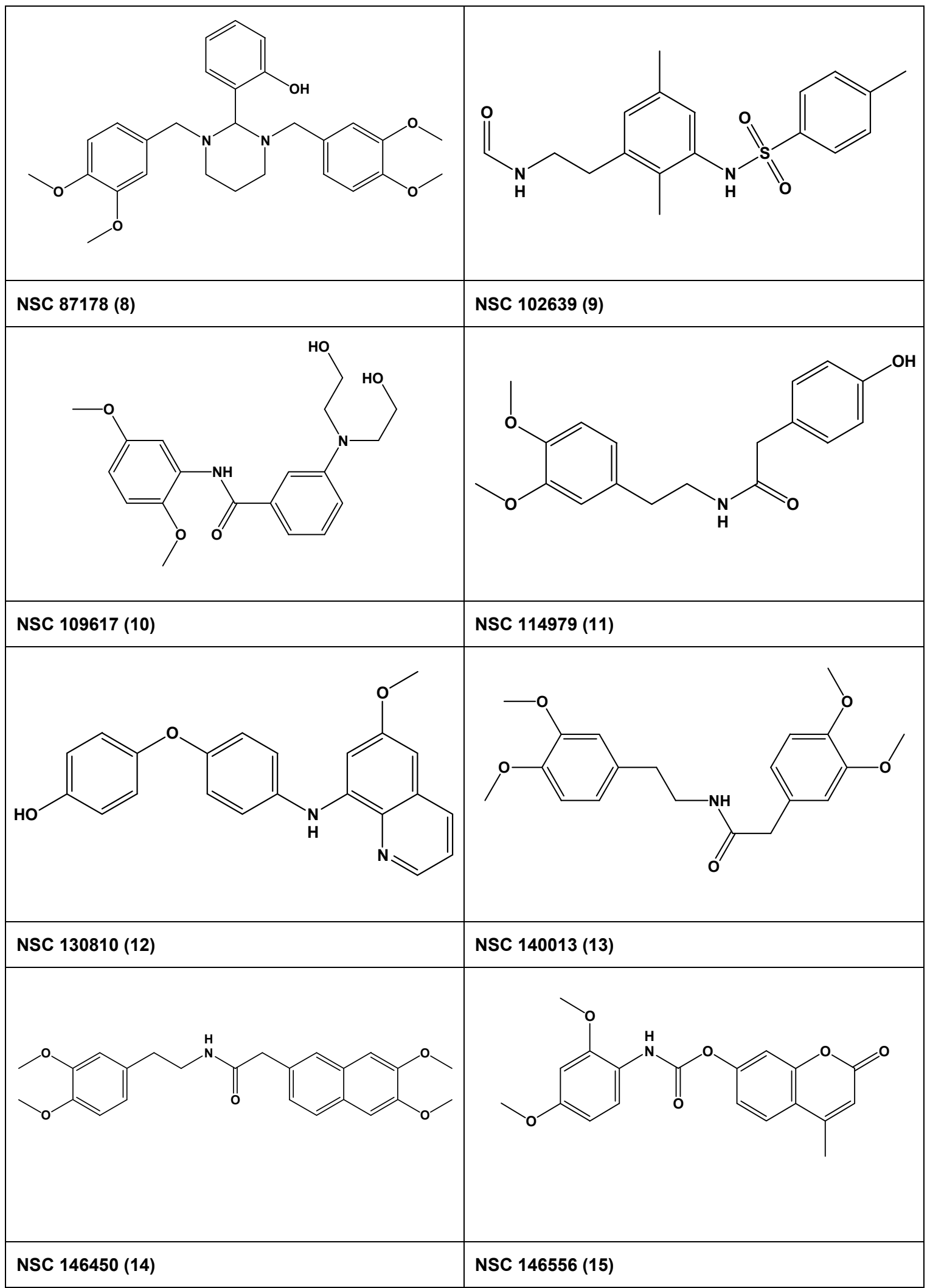

Figure 4: Chemical structures of tBHQ and NSC compounds (8-15) selected for Nrf2 activity 


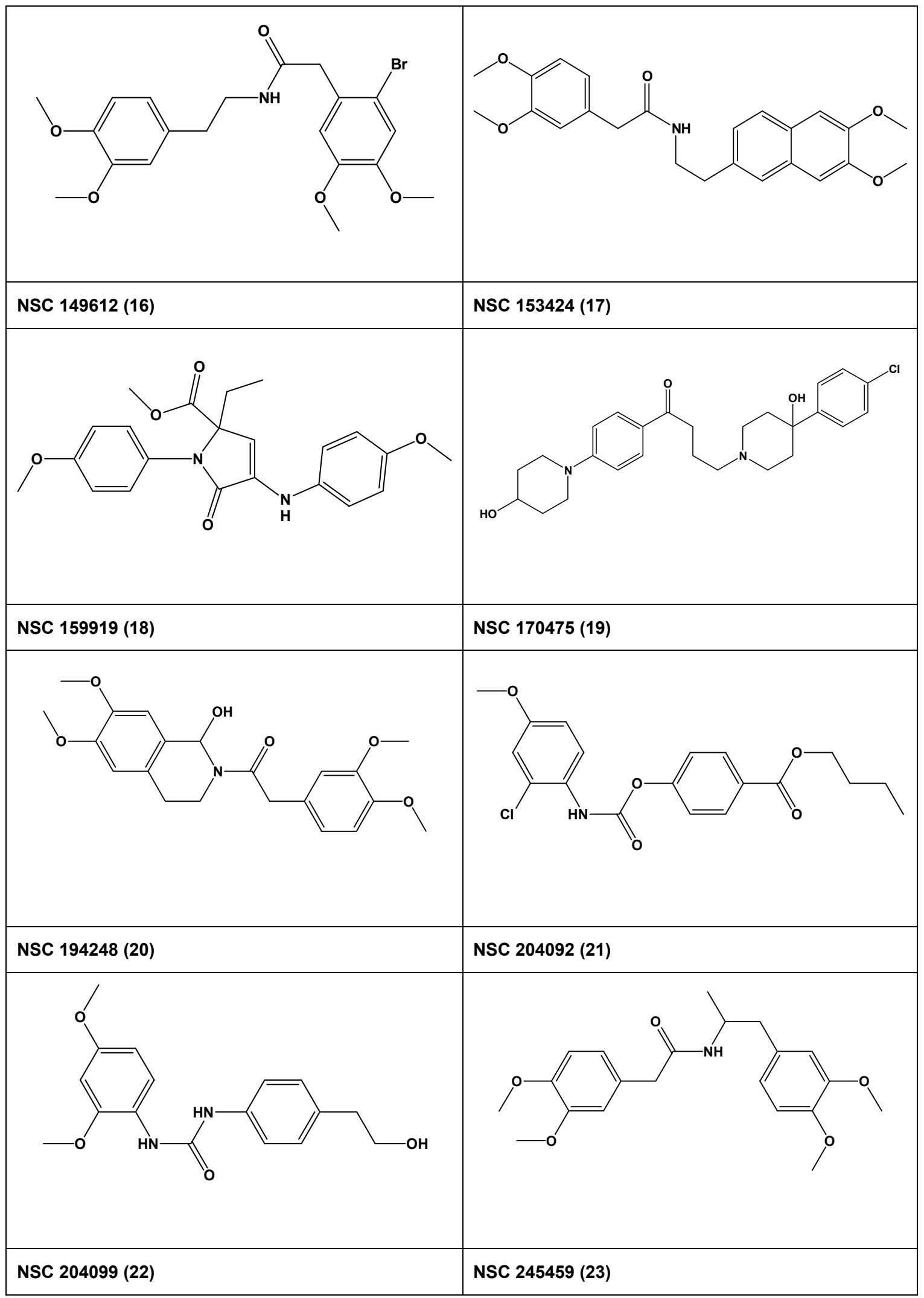

Figure 5: Chemical structures of $\mathrm{tBHQ}$ and NSC compounds (16-23) selected for Nrf2 activity 


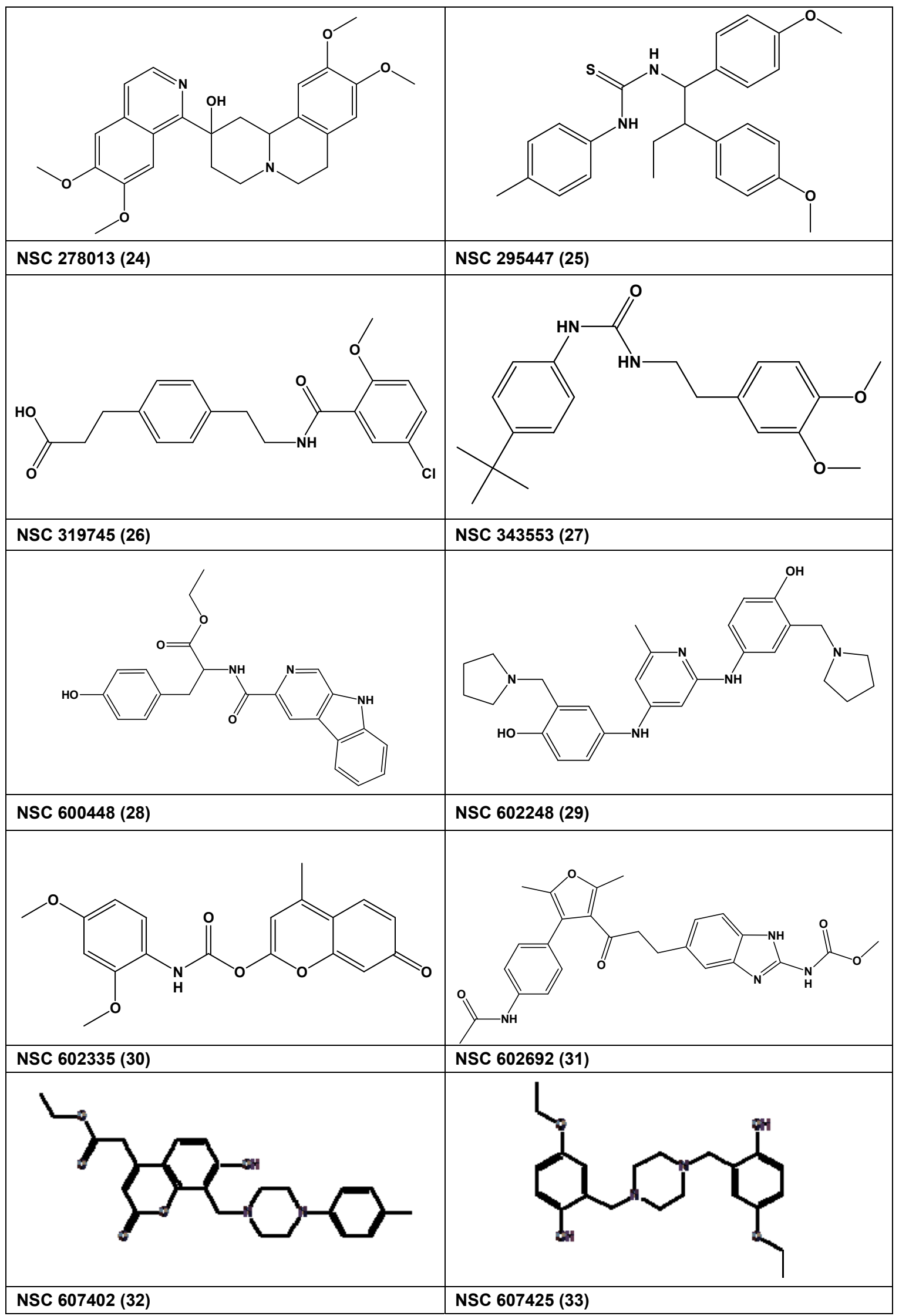

Figure 6: Chemical structures of tBHQ and NSC compounds (24-31) selected for Nrf2 activity 


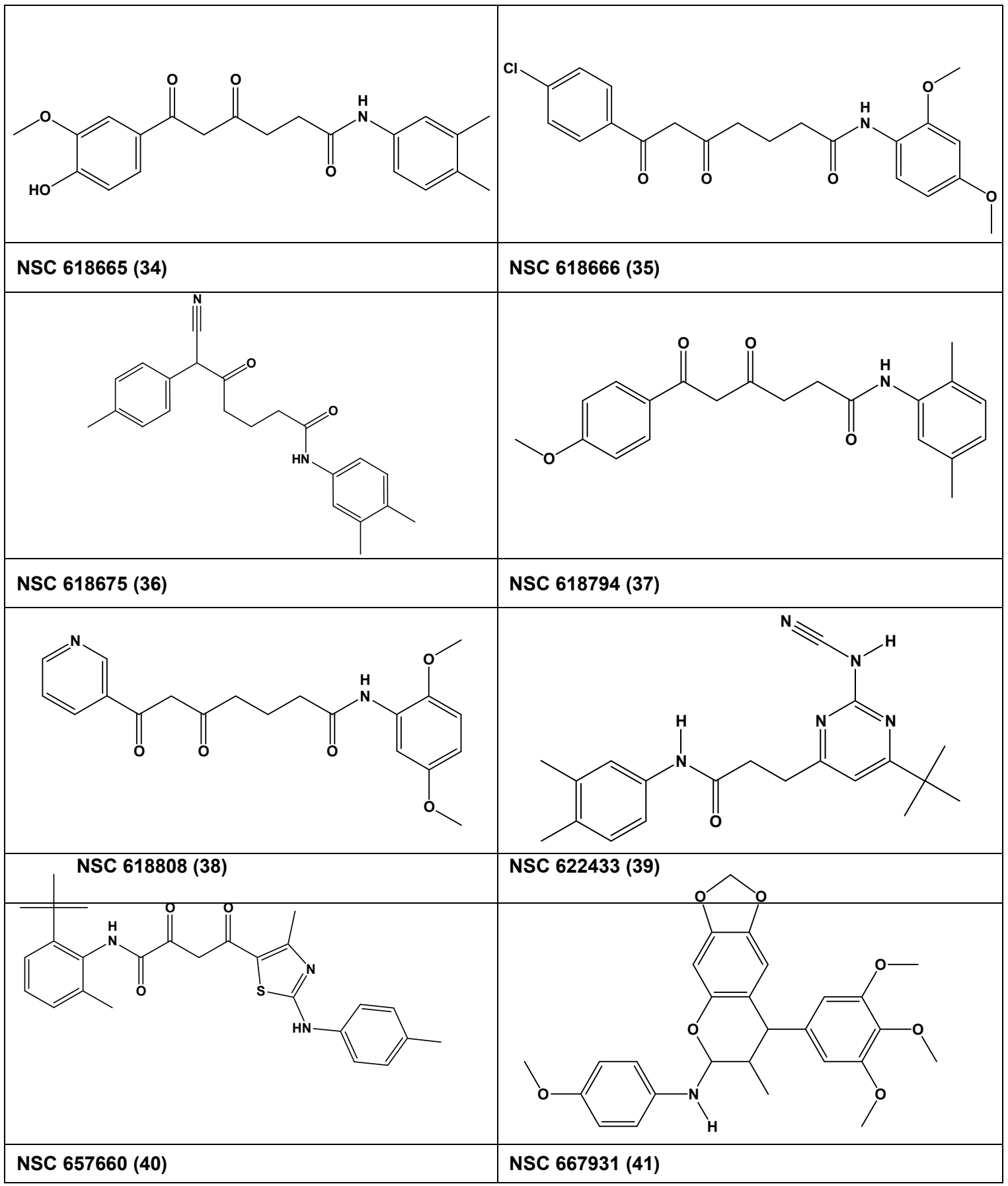

Figure 7: C7hemical structures of tBHQ and NSC compounds (32-41) selected for Nrf2 activity

\section{DISCUSSION}

Many studies have been carried out to find new drugs that can prevent or treat diseases or conditions caused by oxidative stress and/or inflammation. These diseases and conditions include cancer, aging, cardiovascular disease, atherosclerosis, Parkinson's disease, and Alzheimer's disease. Research has demonstrated that Nrf2 activation exerts protective effect against oxidative stress-induced disorders [3, 15]. Thus, Nrf2 is considered an attractive target for the prevention and/or treatment of such diseases.

The transcription factor Nrf2 is encoded by erythroid-derived 2-like2 NFE2L2 gene. The domain structure of Nrf2 contains six Nrf2-ECH homology (Neh) domains. Each Neh domain serves a special function that regulates its 
activation, localization, degradation, and stabilization. It is known that Neh1 is important for the binding of Nrf2 to DNA. Studies have shown that Neh2 and Neh6 are important in targeting Nrf2 for proteasomal degradation by Keap1, while Neh4 and Neh5 domains are important in Nrf2-dependent transactivation [15].

Under normal conditions, the expression of Nrf2 mRNA is constitutively active, but the Nrf2 protein is subjected to continuous proteasomal degradation by complexing with Keap1, a process which is facilitated by its binding to ubiquitin ligase complex [5]. Upon disruption of

Table 4: Inhibition of sphere formation by Nrf2/Keap1 complex inhibitors

\begin{tabular}{lcc}
\hline Compound no. & NSC no. & $\begin{array}{c}\text { Inhibition of } \\
\text { Nrf2 complex } \\
\text { (\%) }\end{array}$ \\
\hline Negative control & DMSO & 30.5 \\
Standard Nrf2 & tBHQ & 100.0 \\
activator & 25446 & 88.3 \\
1 & 41492 & 90.8 \\
2 & 54697 & 111.2 \\
3 & 56626 & 108.0 \\
4 & 71780 & 70.7 \\
5 & 78586 & 79.5 \\
6 & 83410 & 71.7 \\
7 & 87178 & 73.0 \\
8 & 102639 & 72.3 \\
9 & 109617 & 86.7 \\
10 & 114979 & 182.7 \\
11 & 130810 & 94.5 \\
12 & 140013 & 34.1 \\
13 & 146450 & 96.5 \\
14 & 146556 & 66.9 \\
15 & 149612 & 10.7 \\
16 & 153424 & 24.0 \\
17 & 159919 & 49.0 \\
18 & 170475 & 77.0 \\
19 & 194248 & 32.4 \\
20 & 204092 & 80.7 \\
21 & 204099 & 10.8 \\
22 & 245459 & 53.7 \\
23 & 278013 & 18.0 \\
24 & 295447 & 10.6 \\
26 & 319745 & 5.1 \\
27 & 343553 & 25.5 \\
28 & 600448 & 14.1 \\
29 & 602248 & 17.2 \\
30 & 602335 & 0.0 \\
31 & 602692 & 1.1 \\
32 & 607402 & 53.1 \\
33 & 607425 & 73.5 \\
34 & 618665 & 78.6 \\
35 & 618666 & 133.2 \\
37 & 618675 & 37.9 \\
38 & 618794 & 31.8 \\
40 & & 10.3 \\
\hline & 622433 & 47.1 \\
31 & 14.0 \\
\hline & 0.0 \\
\hline
\end{tabular}

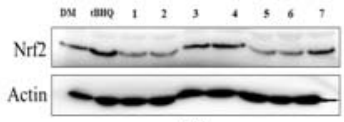

(A)

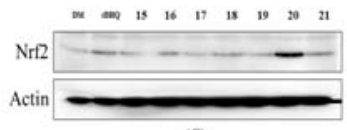

(C)

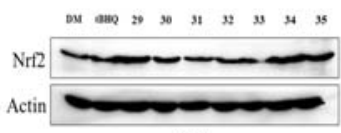

(E)

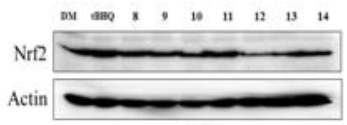

(B)
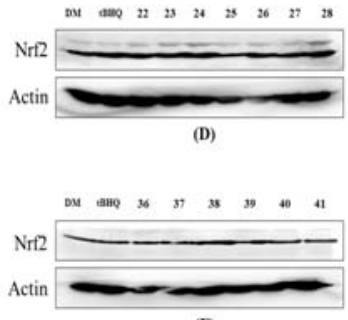

Figure 10: Effect of Nrf2/Keap1 complex inhibitors on Nrf2 expressions in MCF7 cells. The MCF7 cells were treated for $24 \mathrm{~h}$ with DMSO (DM), $30 \mu \mathrm{M}$ of tBHQ, or $30 \mu \mathrm{M}$ of each of the NSC compounds 1-7 (A), (B) 8$14(B), 15-21(C)$, 22-28 (D), 29-35 (E), and 36-41 (F). Thereafter, Nrf2 protein expression was assayed with western blot analysis, with F-actin as loading control

the Nrf2/Keap1 complex by oxidative stress conditions (which affect Neh2 or Neh6 domains), Keap1 is degraded, thereby allowing Nrf2 to build up in the cytosol. After escaping from its complexation with Keap1, Nrf2 migrates to the nucleus where it binds to the AREs or electrophile-response element (EpRE) and induces the expression of antioxidant enzymes [6]. The expressions of the antioxidant enzymes superoxide dismutase, catalase, peroxiredoxin, glutathione peroxidase, $H O-1, N Q O-1$, and GCS, are induced via the Nrf2/Keap1 pathway [16]. The activation of Nrf2 is strongly associated with cytoprotection from the oxidative insults caused by the accumulation of reactive oxygen species (ROS) which are implicated in aging and agerelated diseases [17].

Under normal circumstances, MCF7 is a breast cancer cell line with low levels of Nrf2 protein. However, when treated with $\mathrm{tBHQ}$ and Nrf2activating targets, Nrf2 protein levels are increased, leading to upregulation of the expression of the cytoprotective antioxidant enzyme GCS. Interestingly, 29 out of the 41 tested compounds (about 71\%) produced positive activation of GCS (Figure 4). Compounds 1-4, 11, 12, 14 and 35 produced activation levels similar to, or higher activation than that of the standard Nrf2 activator tBHQ. This underscores the importance of inhibiting the formation of Nrf2/Keap1 complex, as a strategy for stabilizing and activating Nrf2. Another interesting finding is that the effect of Nrf2 activation, and subsequently, GCS activation was effective even after $24 \mathrm{~h}$. This indicates that these compounds might be useful in fighting 
oxidative stress conditions and associated diseases. Most of the tested compounds activated Nrf2.

\section{CONCLUSION}

In the search for new anticancer compounds, many potential targets have been identified in this study using the virtual co-crystallized Nrf2 pharmacophore model. Many of the $\mathrm{NCl}$ samples tested were good inhibitors of Nrf2/Keap1 complexation, and they resulted in disruption of Nrf2 degradation, causing Nrf2 to be stabilized and unregulated. The most interesting result is that Nrf2 activation is long-acting (> $24 \mathrm{~h}$ ). Thus, the disruption of the Nrf2/Keap 1 complex is likely to be an effective cytoprotective approach for combating elevated ROS levels and oxidative stress-related disorders and diseases. Although most of the tested compounds upregulate Nrf2 protein expression, some of these compounds do not upregulate GCS. This can be explained by the fact that Nrf2 is involved in the upregulation of many antioxidant enzymes, not just GCS. However, there is need for further investigations to confirm this.

\section{DECLARATIONS}

\section{Acknowledgement}

The authors thank the National Cancer Institute for their support with $\mathrm{NCl}$ samples. We also thank the Deanship of Scientific Research at the Zarqa University for their generous funds. We are grateful to Ms Doaa AL-Shooli for her assistance in cell culture studies, and to Professor Mahmoud AAl-Sha'er for his assistance in molecular modeling studies.

\section{Conflict of interest}

No conflict of interest is associated with this work.

\section{Contribution of authors}

We declare that this work was done by the authors named in this article and all liabilities pertaining to claims relating to the content of this article will be borne by the authors.

\section{Open Access}

This is an Open Access article that uses a funding model which does not charge readers or their institutions for access and distributed under the terms of the Creative Commons Attribution License (http://creativecommons.org/licenses/by/
4.0) and the Budapest Open Access Initiative (http://www.budapestopenaccessinitiative.org/rea d), which permit unrestricted use, distribution, and reproduction in any medium, provided the original work is properly credited.

\section{REFERENCES}

1. Sasaki H, Shitara M, Yokota K, Hikosaka Y, Moriyama S, Yano M, Fujii Y. Increased NRF2 gene (NFE2L2) copy number correlates with mutations in lung squamous cell carcinomas. Mol Med Rep 2012; 6(2): 391-394.

2. Moi P, Chan K, Asunis I, Cao A, Kan YW. Isolation of NFE2-related factor 2 (Nrf2), a NF-E2-like basic leucine zipper transcriptional activator that binds to the tandem NF-E2/AP1 repeat of the beta-globin locus control region. Proc Natl Acad Sci USA 1994; 91(21): 99269930.

3. Saha S, Buttari B, Panieri E, Profumo E, Saso L. An Overview of Nrf2 Signaling Pathway and its Role in Inflammation. Molecules 2020; 25(22): 5474.

4. Jowsey IR, Jiang $Q$, Itoh $K$, Yamamoto $M$, Hayes JD. Expression of the aflatoxin B1-8,9-epoxide-metabolizing murine glutathione $S$-transferase A3 subunit is regulated by the Nrf2 transcription factor through an antioxidant response element. Mol Pharmacol 200; 64(5): 10181028.

5. Martinez VD, Vucic EA, Thu KL, Pikor LA, Hubaux R, Lam WL. Unique pattern of component gene disruption in the NRF2 inhibitor KEAP1/CUL3/RBX1 E3-ubiquitin ligase complex in serous ovarian cancer. Biomed Res Int 2014; 2014: ID 159459.

6. Giudice A, Arra C, Turco MC. Review of molecular mechanisms involved in the activation of the Nrf2-ARE signaling pathway by chemopreventive agents. In: Higgins P. (eds). Humana Press, Totowa, NJ Transcription Factors. Methods Mol Biol (Methods and Protocols) 2010; 647: 37-74.

7. Al-Sha'er MA, Mansi I, Almazari I, Hakooz N. Evaluation of novel Akt1 inhibitors as anticancer agents using virtual co-crystallized pharmacophore generation. J Mol Graph Model 2015; 62: 213-225.

8. Berman HM, Westbrook J, Feng Z, Gilliland G, Bhat TN, Weissig H, Shindyalov IN, Bourne PE. The Protein Data Bank. Nucleic Acids Res 2000; 28(1): 235-242.

9. Dassault Systèmes. BIOVIA DS. San Diego: Dassault Systèmes; 2016.

10. Users' Manual. Inc. CATALYST_4.11. San Diego, CA.: Accelrys Software; 2005.

11. Protein Data Bank, 2015 [cited 2021 June 01]. Available from: http://www.rcsb.org

12. Venkatachalam CM, Jiang $X$, Oldfield $T$, Waldman $M$. LigandFit: a novel method for the shape-directed rapid docking of ligands to protein active sites. J Mol Graph Model 2003; 21(4): 289-307.

13. Al-Sha'er MA, Taha MO. Application of docking-based comparative intermolecular contacts analysis to validate Hsp90a docking studies and subsequent in silico Trop J Pharm Res, September 2021; 20(9): 1872 
screening for inhibitors. J Mol Model 2012; 18(11): 4843-4863.

14. Turley AE, Zagorski JW, Rockwell CE. The Nrf2 activator tBHQ inhibits $T$ cell activation of primary human CD4 T cells. Cytokine 2015; 71(2): 289-295.

15. Vomund $S$, Schäfer A, Parnham MJ, Brüne $B$, von Knethen A. Nrf2, the Master Regulator of Anti-Oxidative Responses. Int J Mol Sci 2017; 18(12): 2772.
16. Yama K, Sato K, Abe N, Murao Y, Tatsunami R, Tampo $Y$. Epalrestat increases glutathione, thioredoxin, and heme oxygenase-1 by stimulating Nrf2 pathway in endothelial cells. Redox Biol 2015; 4: 87-96.

17. Pawlowska E, Szczepanska J, Koskela A, Kaarniranta K, Blasiak J. Dietary Polyphenols in Age-Related Macular Degeneration: Protection against Oxidative Stress and Beyond. Oxid Med Cell Longev 2019 M; 24: 9682318. 